\title{
Correction to: Establishing Trustworthiness Through Algorithmic Approaches to Qualitative Research
}

Ha Nguyen (D), June Ahn (D), Ashlee Belgrave, Jiwon Lee, Lora Cawelti, Ha Eun Kim, Yenda Prado, Rossella Santagata, and Adriana Villavicencio

\section{Correction to:}

Chapter "Establishing Trustworthiness Through Algorithmic Approaches to Qualitative Research" in: A. R. Ruis and S. B. Lee (Eds.): Advances in Quantitative Ethnography, CCIS 1312, https://doi.org/10.1007/978-3-030-67788-6_4

In the originally published version of the chapter 4, the name of the author was spelled incorrectly. The author's name has been changed as Ashlee Belgrave. 